\title{
Dyspnoea in lupus
}

\author{
Jeremy Fleri Soler, ${ }^{1}$ Andrew Borg, ${ }^{2}$ Cecilia Mercieca ${ }^{2}$
}

'Department of Medicine, Mater Dei Hospital, Msida, Malta

${ }^{2}$ Department of Rheumatology, Mater Dei Hospital, Msida, Malta

\section{Correspondence to} Dr Jeremy Fleri Soler, jeremy.fleri-soler@gov.mt

Accepted 25 October 2017

To cite: Fleri Soler J, Borg A, Mercieca C. BMJ Case Rep Published Online First: [please include Day Month Year]. doi:10.1136/bcr-2017220162

\section{SUMMARY}

A 32-year-old woman suffering from systemic lupus erythematosus presented with a 6-week history of progressive dyspnoea and pleuritic chest pain. Examination was normal apart from reduced air entry at the lung bases.

Arterial blood gases showed hypoxaemia and chest X-ray revealed raised hemidiaphragms without any pleural effusions. Lung function showed a restrictive pathology while high-resolution chest $\mathrm{CT}$ and $\mathrm{CT}$ pulmonary angiogram were negative. Echocardiography showed normal ventricular diameters and no pericardial effusion. Reduced lung volumes and a positive fluoroscopic sniff test lead to a diagnosis of shrinking lung syndrome. Symptoms improved following treatment with glucocorticoids and non-invasive ventilation, but there was no change in lung function.

A year later, our patient presented again with worsening dyspnoea. This time echocardiography revealed severe mitral stenosis with pulmonary hypertension. Mitral valve replacement was performed and dyspnoea resolved. Histology showed Libman-Sachs endocarditis.

\section{BACKGROUND}

We present a case of breathlessness in a patient known to suffer from systemic lupus erythematosus (SLE). Dyspnoea is a common complaint encountered in general medicine with a wide differential diagnosis. Once the common causes were excluded, investigations confirmed that our patient was suffering from shrinking lung syndrome (SLS) - a rare cause of dyspnoea known to occur as a complication of SLE.

However, despite being on treatment for SLS for some time, our patient reported worsening of symptoms. Subsequently, further possible causes were sought and severe mitral stenosis (MS) was found on echocardiography with histology confirming a Libman-Sachs endocarditis.

Although unsure of the chronology of pathologies, the two diagnoses likely overlapped with our patient suffering from dyspnoea secondary to both SLS and MS. Such a case highlights the importance of searching for possible alternative diagnoses in cases of relapse of symptoms or when treatment is no longer effective.

\section{CASE PRESENTATION}

A 32-year-old woman known to suffer from SLE presented with a 6-week history of insidious, progressive breathlessness, orthopnoea, a dry cough and pleuritic chest pain but no fever, haemoptysis or lower limb swelling. She also reported fatigue, poor sleep and morning headaches.
She was diagnosed with SLE 5 years previously, on the basis of a malar rash, arthritis, oral ulcers, serositis and anaemia together with the presence of a strongly positive antinuclear antibody, anti-ds DNA and anti-Sm antibodies. She also had a history of pre-eclampsia and diffuse proliferative lupus nephritis. She was taking mycophenolate sodium, hydroxychloroquine and low-dose prednisolone (5 mg daily). She did not smoke and had no history of occupational exposure to lung allergens or pets and no recent long haul flights.

Cardiovascular and neurological examination was normal and she was afebrile. The respiratory rate was 17 breaths per min. Respiratory examination revealed decreased breath sounds at the lung bases. There were no rashes, mouth ulcers or evidence of synovitis.

\section{INVESTIGATIONS}

Full blood count, creatine kinase and complement levels were normal. C reactive protein was $24 \mathrm{mg} / \mathrm{L}$ (N.V. $\quad 0-6 \mathrm{mg} / \mathrm{L}$ ) while erythrocyte sedimentation rate was $54 \mathrm{~mm} /$ hour. Arterial blood gases on air were compatible with relative hypoxaemia; oxygen saturation was $96 \%, \mathrm{PaO}_{2} 75 \mathrm{~mm} \mathrm{Hg}$ and $\mathrm{PaCO}_{2} 33 \mathrm{~mm} \mathrm{Hg}$. ECG was normal and echocardiography showed thickening and calcification of the posterior leaflet of the mitral valve with mild mitral regurgitation but normal left ventricular (LV) size and function and no pericardial effusion. Figure 1 depicts the chest X-ray (CXR) taken on admission, showing a right elevated hemidiaphragm and reduced lung volumes but no consolidation, effusions or pulmonary infiltrates and the cardiac shadow was normal.

Lung function tests showed a restrictive lung pattern (forced expiratory volume in one second $\left(\mathrm{FEV}_{1}\right.$ ): forced vital capacity (FVC) 83, FVC $54 \%$ predicted, vital capacity $62 \%$ predicted and total lung capacity $72 \%$ predicted) and a reduced diffusing lung capacity (DLCO). High-resolution CT (HRCT) of the chest and CT pulmonary angiogram (CTPA) were normal excluding interstitial lung disease and pulmonary thromboembolism respectively. Fluoroscopic sniff test showed bilateral paradoxical elevation of the paralysed diaphragm on inspiration and no movement on expiration, in keeping with bilateral diaphragmatic paralysis-SLS. Given a diagnosis of SLS in the context of SLE, the patient was treated with supportive ventilation Bilevel Positive Airway Pressure (BiPAP) and immunosuppression with glucocorticoids and rituximab. 


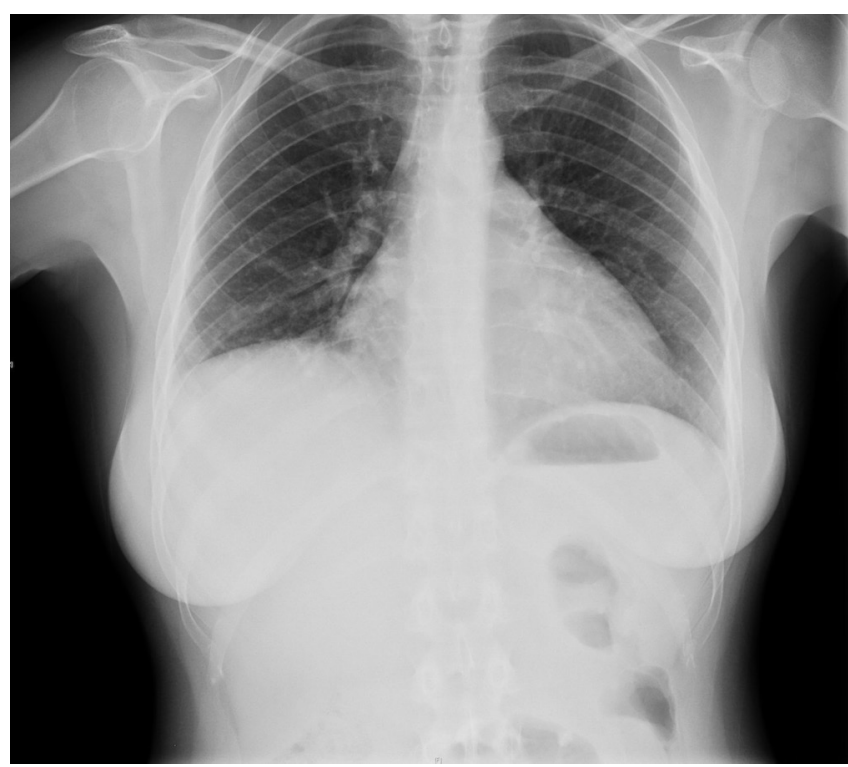

Figure 1 Chest X-ray on admission showing a raised right hemidiaphragm.

\section{DIFFERENTIAL DIAGNOSIS}

Breathlessness in SLE presents a diagnostic challenge because of the wide differential diagnosis. Table 1 lists possible aetiologies of dyspnoea in lupus. Pleuropulmonary manifestations of SLE are manifold and each may present with dyspnoea as the only presenting complaint. Cardiac involvement is also common and must also be considered in the differential diagnosis. ${ }^{12}$

Pneumonia is very common in patients suffering from SLE. Immunosuppression from chronic disease and treatment predisposes patients to both community acquired and atypical/opportunistic infections. ${ }^{1}$ Our patient was afebrile and examination and investigations including CXR were not suggestive of an underlying infection.

Up to $60 \%$ of patients with SLE have pleuritis with or without a pleural effusion, which can be unilateral or bilateral. Typically patients present with dyspnoea, chest pain and at times a cough. ${ }^{3}$ Clinically, our patient had reduced air entry on chest examination. However, pleural effusions were excluded on imaging.

In spite of the insidious history, pulmonary thromboembolism needed to be considered. SLE is a thrombophilic disorder predisposing to chronic emboli into the pulmonary circulation with subsequent pulmonary hypertension. ${ }^{1}$ Approximately 20\% of patients with SLE are said to have clinically significant levels of antiphospholipid antibodies causing a secondary antiphospholipid syndrome (APS), predisposing patients to thromboembolism. ${ }^{4}$ In this case, APS antibodies and lupus inhibitor screen were negative

Table 1 Causes of dyspnoea in systemic lupus erythematosus

\begin{tabular}{ll}
\hline Pleuropulmonary causes & Pneumonia \\
& Pleuritis with or without effusion \\
& Acute lupus pneumonitis \\
& Chronic interstitial lung disease \\
& Pulmonary embolism \\
& Shrinking lung syndrome \\
& Pulmonary hypertension \\
\hline Cardiac causes & Pericarditis with or without effusions \\
\hline & Non-infective endocarditis \\
\hline & Myocarditis \\
\hline
\end{tabular}

making APS unlikely while CTPA and echocardiography showed no evidence of pulmonary embolism or hypertension.

Interstitial lung disease could have explained the restrictive pattern. Involvement of the lung parenchyma can present acutely as in the case of lupus pneumonitis or chronically as a result of non-specific interstitial fibrosis. ${ }^{5}$ In the latter case, the restrictive lung function pattern will be accompanied by a reduced DLCO. ${ }^{1}$ However, the normal HRCT excluded interstitial lung disease.

Having excluded the the most common causes for dyspnoea and chest pain in SLE and having found a restrictive pattern and raised hemidiaphragms, SLS was considered. This was supported by a positive fluoroscopic Sniff Test. SLS is a rare complication of SLE characterised by bilaterally reduced motion of diaphragm domes. ${ }^{6}$

\section{TREATMENT}

Treatment options for SLS are based on case reports. A literature review found that $95 \%$ of patients were given glucocorticoids. ${ }^{6}$ Just over $50 \%$ of patients were given additional immunosuppressive agents, the most popular were azathioprine and cyclophosphamide while methotrexate and mycophenolate mofetil have also been used with variable outcomes; however, more recently, a number of cases have been treated successfully with rituximab. ${ }^{6}$

After the initial diagnosis of SLS, our patient was treated with high-dose oral prednisolone and ventilatory BIPAP (IPAP $10 \mathrm{~cm} \mathrm{H}_{2} \mathrm{O}$, Expiratory positive airway pressure $5 \mathrm{~cm} \mathrm{H}_{2} \mathrm{O}$ and oxygen flow rate of $16 / \mathrm{min})$. Repeat blood gases 1 hour after commencement of BiPAP showed normal blood gases with full oxygen saturation of $99 \%$. After a few days of nocturnal BiPAP therapy, there was a marked improvement of her chest pain, orthopnoea, headaches and morning fatigue. Further immunosuppression was attempted with rituximab. Unfortunately, this had to be stopped following a severe allergic rash during the second dose of rituximab.

At 1 year, our patient reported worsening breathlessness on mild exertion, despite compliance with BiPAP and immunosuppression with prednisolone and mycophenolate. Her lung function had deteriorated further and blood tests revealed low complement levels. There were no other signs suggestive of a pulmonary cause and CXR showed no evidence of pneumonia or pleural effusion.

Echocardiography at this point showed thickened mitral valve (MV) leaflets with diminished opening in keeping with severe MS and moderate Tricuspid regurgitation suggesting pulmonary hypertension. Figure 2 illustrates the 4-chamber view with colour Doppler and figure 3 depicts the Doppler values,

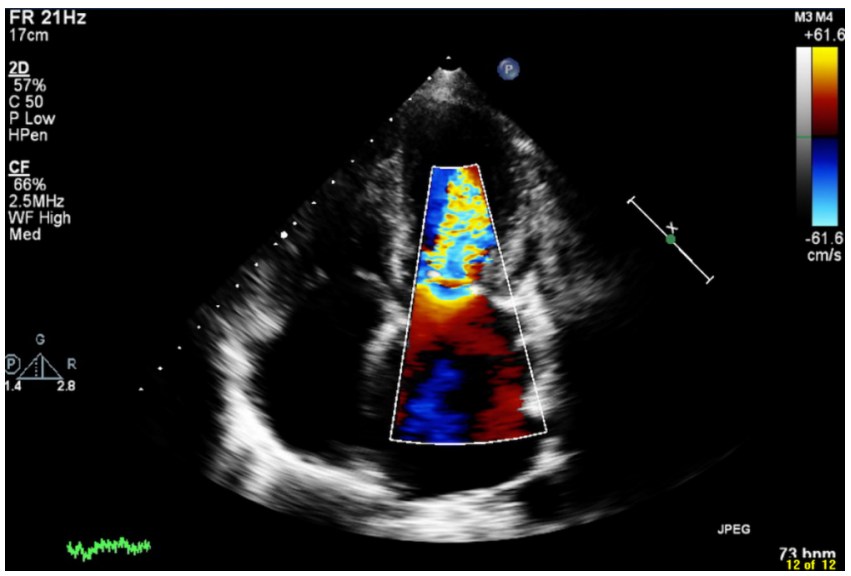

Figure 2 Echocardiogram 4-chamber view with colour Doppler showing mitral stenosis. 


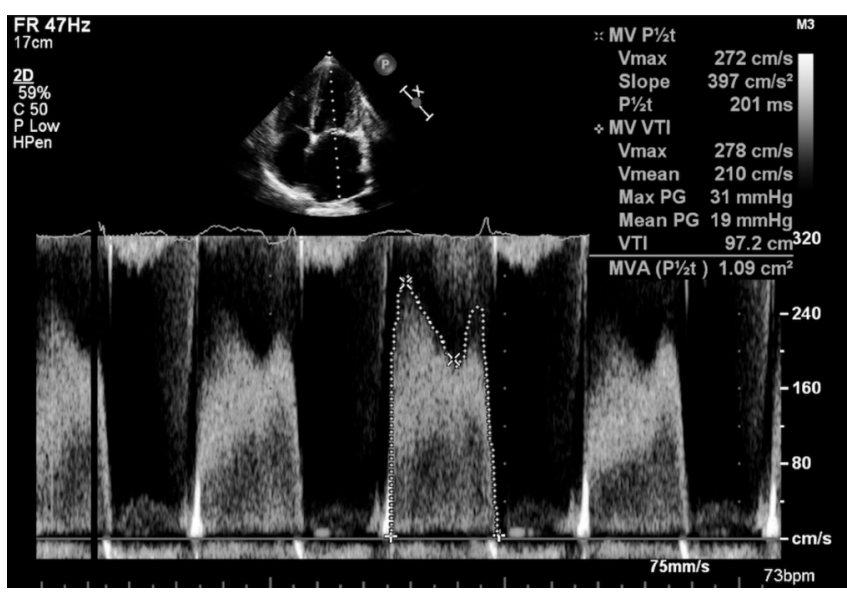

Figure 3 Echocardiogram with Doppler values. Mean pressure gradient of $19 \mathrm{~mm} \mathrm{Hg}$ qualifying mitral stenosis as severe.

with a mean pressure gradient of $19 \mathrm{~mm} \mathrm{Hg}$ supporting a diagnosis of severe MS. Angiography and right heart studies were carried out, which confirmed pulmonary hypertension (PA pressure $90 / 45 \mathrm{~mm} \mathrm{Hg}$ ) and significant MS.

Because of this deterioration and signs of increased activity of SLE, our patient was treated with cyclophosphamide as an alternative to rituximab and referred for urgent mitral valve replacement. Anticoagulation was started postoperatively.

\section{OUTCOME AND FOLLOW-UP}

The mitral valve replacement was a very challenging one due to very dense adhesions around the valve and endocardium. Resected tissue was sent for histology, which confirmed LibmanSacks endocarditis.

Six months following the mitral valve replacement (MVR), our patient reported better exercise tolerance. Interestingly, as depicted in table 2, there was an improvement in lung function including DLCO, such that she no longer required nocturnal BiPAP. The improvement of the latter is probably a result of the cyclophosphamide.

A cardiac MRI was performed 10 months postop to rule out a pancarditis. It showed global pericardial inflammation but no myocardial oedema. The pericarditis may have been postpericardiotomy syndrome post-MVR.

\section{DISCUSSION}

This is a complex case that deals with a dual pathology of SLS and Libman-Sacks endocarditis. Both are serious complications, which commonly present with shortness of breath and chest

Table 2 Change in lung function over time

\begin{tabular}{|c|c|c|c|}
\hline$\%$ Predicted & $\begin{array}{l}2011 \text {-on } \\
\text { admission }\end{array}$ & $\begin{array}{l}2012 \text {-after an } \\
\text { incomplete course of } \\
\text { rituximab, reporting } \\
\text { worsening dyspnoea }\end{array}$ & $\begin{array}{l}\text { 2015-postmitral } \\
\text { valve replacement }\end{array}$ \\
\hline FVC & 54 & 57 & 63 \\
\hline $\mathrm{FEV}_{1}$ & 53 & 53 & 67 \\
\hline $\mathrm{FEV}_{1}: \mathrm{FVC}$ & 84 & 82 & 83 \\
\hline VC & 62 & 58 & 63 \\
\hline TLC & 76 & 60 & 76 \\
\hline DLCO & 57 & 63 & 80 \\
\hline
\end{tabular}

DLCO, diffusing lung capacity; FEV , forced expiratory volume in one second; FVC, forced vital capacity; TLC, total lung capacity; VC, vital capacity. pain yet require different management. Timely diagnosis and management is key to prevent irreversible damage.

SLS has an incidence of $1 \%$ in the SLE population. The pathophysiology is unclear but a number of mechanisms have been suggested including neuropathic or myopathic processes possibly as a result of deposition of immune complexes and ischaemic lesions. Previous episodes of pleuritis could also lead to formation of adhesions, diaphragmatic splinting and dysfunction. Because it is rare, diagnosis is often missed or delayed as physicians search for other causes of dyspnoea. As already discussed, management is largely based on case series and includes glucocorticoids and immunosuppressive agents such as azathioprine, cyclophosphamide and, more recently, rituximab. ${ }^{6}$ Eighteen per cent of patients return to baseline function with no functional sequelae while $80 \%$ showed mild/moderate improvement in pulmonary function test (PFTs) but persistence of a restrictive defect. $^{6}$

Goswami et al describe a case of newly diagnosed SLE that in addition to presentation of constitutional symptoms also described persistent dyspnoea and even needed critical care admission for ventilation. Glucocorticoid therapy did not help but the patient improved with rituximab and by the second infusion she had returned to her baseline function, with some residual restrictive lung function. ${ }^{8}$

We do not think that significant MS was the cause of the shortness of breath at the initial presentation as the patient underwent an echocardiogram at the time. Although endocarditis may have been present at that time, there was no evidence of functional compromise. Endocarditis in SLE is common and present in up to $11 \%$ of patients. ${ }^{9}$ Libman-Sacks endocarditis is a non-infective, verrucous endocarditis of valve leaflets, papillary muscles and mural endocardium. Lesions typically consist of immune complexes and mononuclear cells. It is thought that immunological insults result in valvular oedema leading to diffuse valve thickening, with or without vegetations and eventual scarring and dysfunction of the valve ultimately resulting in regurgitation and/or stenosis. ${ }^{2}$ Libman-Sacks lesions are commonly associated with primary or secondary APS. However, as the pathogenesis of Libman-Sacks endocarditis is still unclear, valvular lesions have also been described in SLE regardless of antiphospholipid antibody positivity as in our case.'

Libman-Sacks endocarditis most commonly affects the mitral valve followed by the aortic valve, but any of the cardiac valves may be involved. It is typically asymptomatic but may result in severe complications such as bacterial endocarditis, thromboembolic events (eg, stroke, transient ischaemic attack), severe valvular regurgitation and/or stenosis requiring surgery. Interestingly, Eric et al describe a case of Libman-Sacks endocarditis affecting a bioprosthetic valve. This patient required MVR with a bovine bioprosthetic valve at a young age for what was thought to be rheumatic heart disease. He later presented with dyspnoea and echocardiography revealed MS with severely raised pulmonary pressures. A redo valve replacement with a mechanical valve was performed. Histology revealed Libman-Sacks endocarditis and serology confirmed SLE. ${ }^{10}$

Management of Libman-Sacks is debatable and includes immunosuppression, anticoagulation (thromboembolic prophylaxis), endocarditis prophylaxis and heart failure treatment if symptomatic. Ultimately some patients may even require valve surgery (1\%-8\%). Based on case reports, stenosis should be treated surgically either by repair or replacement of the MV. ${ }^{11}$ In this case, given the severity of MS, valve replacement was definitely indicated. The treatment with cyclophosphamide was admittedly debatable. However, given the progressive and 
aggressive course of SLE in this patient, it was felt that stronger immunosuppression was required and the benefits of treatment would outweigh the risks.

To our knowledge, this is the first case published that discusses a dual aetiology of dyspnoea having SLE as the overall entity. SLE is a complex multisystem disease. It is important to keep an open mind when managing unremitting or progressive symptoms and search for alternative or additional diagnosis.

\section{Learning points}

- Patients with lupus presenting with shortness of breath need to be thoroughly investigated. Rare causes such as shrinking lung syndrome and mitral stenosis complicated by pulmonary arterial hypertension need to be kept in mind.

- While immunosuppression is the mainstay of treatment for active systemic lupus erythematosus (SLE), different pathologies need specific therapies such as surgery.

- Valvular involvement is common in SLE and a low threshold for echocardiography is warranted.

Contributors All authors contributed to the production of this case report. JFS worked on research and initial write-up. AB and CM directly worked on the concept of the case report as well as repeat corrections and final critique on the paper.

Competing interests None declared.

Patient consent Obtained.
Provenance and peer review Not commissioned; externally peer reviewed.

(C) BMJ Publishing Group Ltd (unless otherwise stated in the text of the article) 2017. All rights reserved. No commercial use is permitted unless otherwise expressly granted.

\section{REFERENCES}

1 Crestani B. The respiratory system in connective tissue disorders. Allergy 2005:60:715-34

2 Miner JJ, Kim AH. Cardiac manifestations of systemic lupus erythematosus. Rheum Dis Clin North Am 2014;40:51-60.

3 Lynch DA. Lung disease related to collagen vascular disease. J Thorac Imaging 2009;24:299-309.

4 Ünlü O, Zuily S, Erkan D. The clinical significance of antiphospholipid antibodies in systemic lupus erythematosus. Eur J Rheumatol 2016;3:75-84.

5 Fidler L, Keen KJ, Touma Z, et al. Impact of pulmonary disease on patient-reported outcomes and patient-performed functional testing in systemic lupus erythematosus. Lupus 2016;25:1004-11.

6 Borrell H, Narváez J, Alegre JJ, et al. Shrinking lung syndrome in systemic lupus erythematosus: A case series and review of the literature. Medicine 2016;95:e4626.

7 Toya SP, Tzelepis GE. Association of the shrinking lung syndrome in systemic lupus erythematosus with pleurisy: a systematic review. Semin Arthritis Rheum 2009;39:30-7.

8 Goswami RP, Mondal S, Lahiri D, et al. Shrinking lung syndrome in systemic lupus erythematosus successfully treated with rituximab. OJM 2016;109:617-8.

9 Moyssakis I, Tektonidou MG, Vasilliou VA, et al. Libman-Sacks endocarditis in systemic lupus erythematosus: prevalence, associations, and evolution. Am J Med 2007; 120:636-42.

10 Sladek EH, Accola KD. Antiphospholipid syndrome and libman-sacks endocarditis in a bioprosthetic mitral valve. Ann Thorac Surg 2016;101:e29-e31.

11 Foroughi M, Hekmat M, Ghorbani M, et al. Mitral valve surgery in patients with systemic lupus erythematosus. ScientificWorldJournal 2014;2014:1-9.

Copyright 2017 BMJ Publishing Group. All rights reserved. For permission to reuse any of this content visit

http://group.bmj.com/group/rights-licensing/permissions.

BMJ Case Report Fellows may re-use this article for personal use and teaching without any further permission.

Become a Fellow of BMJ Case Reports today and you can:

- Submit as many cases as you like

- Enjoy fast sympathetic peer review and rapid publication of accepted articles

- Access all the published articles

- Re-use any of the published material for personal use and teaching without further permission

For information on Institutional Fellowships contact consortiasales@bmjgroup.com

Visit casereports.bmj.com for more articles like this and to become a Fellow 